\title{
Diffusion of single long polymers in fixed and low density matrix of obstacles confined to two dimensions
}

\author{
Ryuzo Azuma and Hajime Takayama \\ Institute for Solid State Physics, The university of Tokyo \\ 7-22-1 Roppongi, Minato-ku, Tokyo 106-8666, Japan
}

(August 2, 2018)

83.10.Nn, $61.25 . \mathrm{Hq}$

Diffusion properties of a self-avoiding polymer embedded in regularly distributed obstacles with spacing $a=20$ and confined in two dimensions is studied numerically using the extended bond fluctuation method which we have developed recently. We have observed for the first time to our knowledge, that the mean square displacement of a center monomer $\phi_{M / 2}(t)$ exhibits four dynamical regimes, i.e., $\phi_{M / 2}(t) \sim t^{\nu_{m}}$ with $\nu_{m} \sim 0.6,3 / 8,3 / 4$, and 1 from the shortest to longest time regimes. The exponents in the second and third regimes are well described by segmental diffusion in the "self-avoiding tube". In the fourth (free diffusion) regime, we have numerically confirmed the relation between the reptation time $\tau_{d}$ and the number of segments $M, \tau_{d} \propto M^{3}$.

\section{INTRODUCTION}

The reptation theory, proposed for concentrated solution of linear polymers [1], is based on the assumptions that a polymer moves in a "tube" made of the other polymers and that the tube does not deform till the polymer of interest creeps out of it. Since the theory was proposed, various investigations have been done on dynamic properties of a polymer in networks of immobile obstacles. As for the case of concentrated solution, Baumgärtner et al [2] studied dynamics of a freely jointed chain with the Lennard-Jones potential in a dense network of frozen-in chains. Evans and Edwards [3], on the other hand, proposed an efficient lattice model with stochastic local jump motion of segments in regularly distributed obstacles on the cubic lattice, which simulates such phenomena as dynamics of a DNA molecule in agarose gel.

Study of diffusion property of a single real polymer (self-avoiding chain) in fixed and low density network of obstacles is of importance, since details of DNA dynamics in agarose gel, etc. [4 6] have been studied by many experimental works along with recent developments in technical aspects 7,8]. To see entanglement effect, investigations of a self-avoiding polymer in two dimensions would be desired. In two dimensions, a molecule with relatively low polymerizations can entangle with many obstacles since it extends larger than a polymer in three dimensions.
Recently, Maier and Rödler [9] observed conformation and diffusion of a single DNA molecule electrostatically bound to fluid cationic lipid bilayers by fluorescence microscopy. They measured radius of gyration $R_{I}$ and obtained the relation $\left\langle R_{I}^{2}\right\rangle \sim N^{2 \nu}$ where $N$ is the number of base pairs of the molecule and $\nu=0.79 \pm 0.04$. For diffusion coefficient of the center of mass $D_{G}$ they showed $D_{G} \propto N^{-1}$. These results agree well with the theoretical predictions for a self-avoiding polymer in two dimensions without hydrodynamic interactions. It seems feasible to arrange obstacles on that layer and somehow measure displacement of a particular part of DNA to examine diffusion property of a self-avoiding polymer in a fixed network of obstacles in two dimensions.

In the present work, dynamic properties of a single polymer in regularly distributed obstacles in two dimensions is studied by means of the extended bond fluctuation method (e-BFM) which we have developed recently $[10]$. From the simulated mean square displacement of a center monomer $\phi_{M / 2}(t)$, we can distinguish clearly four dynamical regimes, as is the case of a Gaussian chain [11] in the same network of obstacles. The simulated exponents $\nu_{m}$ of $\phi_{M / 2}(t) \sim t^{\nu_{m}}$ are $\nu_{m} \sim 0.6$, $3 / 8,3 / 4$, and 1 from the shortest to longest time regimes. The exponents in the early three regimes differ from those of the Gaussian chain, but they are well described when the excluded volume effect is taken into account. In particular, dynamics in the second and third regimes is interpreted as segmental diffusion in the "self-avoiding tube," where displacement $\Delta s$ along that tube corresponds to $a \Delta s^{\nu}$ in real space with $a$ and $\nu$ being the tube diameter and the exponent relating radius of inertia $R_{I}$ and the number of segments $M$ as $R_{I} \sim M^{\nu}$, respectively. The reptation time $\tau_{d}$, as for the crossover time between the third and fourth (free diffusion) regimes, is ascertained to be proportional to $M^{3}$ with $M$ being the number of segments of the chain.

In the next section we explain the details of the simulational methodology and parameters which we have used. The scaling theory of tube model for a self-avoiding polymer in the network of fixed and low density obstacles is described in the Sec. III. We then present the results and their scaling analyses compared to the theory in Sec. IV, and conclude them in the final section. 


\section{METHOD}

The bond fluctuation method (BFM) 12,13, is known to provide effective Monte Carlo (MC) algorithm to study static and dynamic properties of various phenomena involved in polymers 14 . We have adopted a square lattice version of the method where a polymer is described by a sequence of monomers which occupies four sites of the smallest square cell. The monomers are not allowed to occupy the same site (excluded volume interaction or effect (EVE)) and connected by bonds which can vary its length $l$ in the range $2 \leq l \leq \sqrt{13}$. The elemental MC step is a trial of displacement of each monomer by one lattice spacing to a randomly chosen direction. The process satisfies the self-avoiding condition.

The extended-BFM which we have proposed [10] and used in the present study incorporates a non-local movement of the 's-monomer' into the BFM. The s-monomer is defined as the one whose nearest neighbors separate not greater than $\sqrt{13}$ with each other. An s-monomer can be displaced to any monomer pairs between the nearest s-monomers by a stochastic process which fulfills detailed balance condition. This new non-local process can be introduced in any relative frequency into the conventionalBFM above mentioned. We have used the following combined process: at even (odd) time step of the c-BFM, the new process for even (odd) numbered s-monomers is tried.

The e-BFM has been initially introduced to overcome the trapping or pinning difficulty which the c-BFM is faced in gel electrophoresis under a large electric field. It has turned out that the method reproduces static and dynamic properties also under a vanishing field correctly, and more faster than the c-BFM for polymer dynamics in a space with fixed obstacles. In fact, the ratio $A$ of the diffusion constants obtained by the c-BFM and eBFM is less than unity: $A \sim 1 / 2$ and $1 / 3$ for $a=\infty$ (no obstacles) and 20, respectively, with $a$ being the distance between the obstacles.

By means of the e-BFM we here mainly observed the mean square displacement of the center monomer $\phi_{M / 2}(t)$ as a representative of inner monomers. The initial configurations are provided by the self-avoiding walk algorithm where monomer sequence is made by iteration of trials of making a new end. If it finds bond crossing or monomer overlap, it stops making the chain and go to another starting position. Although this algorithm can give independent equilibrium configurations, the averaged time needed for a complete configuration turned out to be quite large due to the low acceptance rate for each trial. So we have relaxed the condition for accepting a new end as that it allows up to $\omega$ trials. Here we have chosen $\omega=7 \sim 14$. The number of samples given at $t=0$, the start time of the idle run, was $512 \sim 788$. The interval of the measuring run $t_{f}-t_{i}$ was taken so as to $\left[\phi_{M / 2}\left(t_{f}-t_{i}\right)\right]^{1 / 2} \gtrsim C R_{I}$, with $C \gtrsim 2$. The system size was $3 M \times 3 M$ in which even a fully extended polymer cannot interact with itself through the periodic boundary conditions introduced. We distributed, regularly in a spacing $a=20$, obstacles of a unit square cell, with which any monomer site cannot overlap (the EVE).

Let us here compare some results simulated by the cBFM and the e-BFM. As an example, we show $\phi_{M / 2}(t)$ for $a=20$ and $M=150$ in Fig. 1. At $t<\tau_{e}, \tau_{e}$ being the crossover time between regimes I and II introduced in the next section, $\phi\left(t / A_{\infty}\right)$ with $A_{\infty} \cong 0.5$ simulated by the c-BFM and $\phi_{M / 2}(t)$ by the e-BFM coincide with each other, and exhibit the power-law growth which is proportional to $t^{0.6}$. The latter is the characteristics of monomer diffusion in free space. At $t>\tau_{e}$, on the other hand, where monomer diffusion is affected by the surrounding obstacles, $\phi_{M / 2}(t)$ by the e-BFM is seen to coincide with $\phi_{M / 2}\left(t / A_{20}\right)$ with $A_{20} \cong 0.3$ by the c-BFM. The factors $A_{\infty}$ and $A_{20}$ are just the ratios $A$ of the diffusion constants mentioned above. Thus the results shown in Fig. 1 demonstrates that, there is no fundamental difference in diffusion properties of the polymer simulated by the c-BFM and the e-BFM.

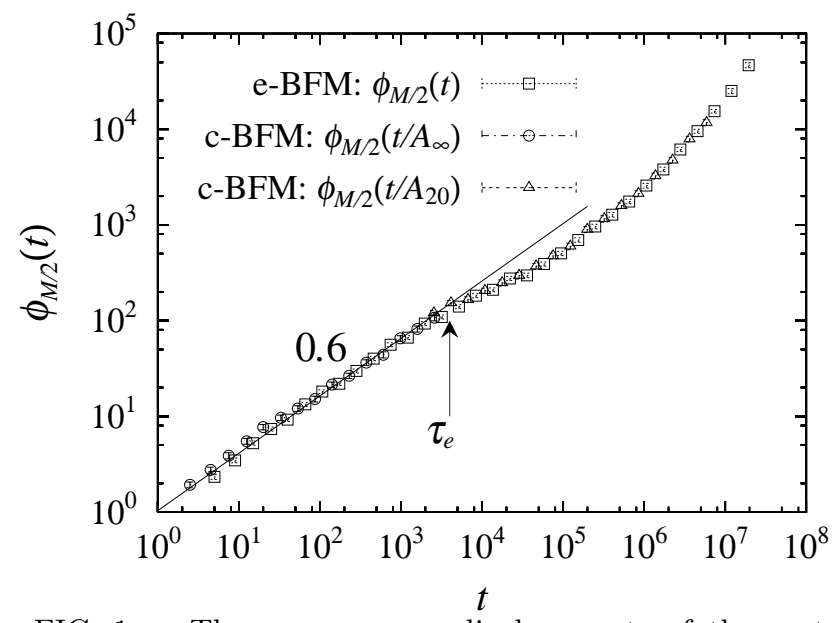

FIG. 1. The mean square displacements of the center monomer of the $M=150$ chain in regular distributed (spacing $a=20$ ) obstacles. The symbols $\square, \bigcirc$, and $\triangle$ are of the e-BFM, c-BFM for $t<\tau_{e}$, and c-BFM for $t>\tau_{e}$ respectively.

\section{SCALING THEORY}

We begin with brief summary of the scaling argument on the tube theory of a Gaussian chain moving in a network of fixed obstacles [11]. There exist four time regimes in which the chain exhibits different dynamics. In the earliest regime denoted by regime I, the $n$-th inner monomer fluctuates without perceiving wall of the tube, and its mean square displacement $\phi_{n}(t)$ is written as $\phi_{n}(t) \sim t^{\nu_{m}}$ with $\nu_{m}=1 / 2$ 15]. Here we put $\zeta / k_{B} T=1$ with $\zeta$ being the friction coefficient of a segment and the segment length $b$ unity. In regimes II and III, the polymer motion perpendicular to the tube coordinate is restricted. Diffusional displacement of the prim- 
itive chain along the tube $\left\langle\Delta s^{2}\right\rangle$ in a time interval $t$ is written as $\left\langle\Delta s^{2}\right\rangle \sim a t^{1 / 2}$ and $a t^{1}$ before (regime II) and after (regime III) the Rouse relaxation time $\tau_{r} \sim M^{2}$, where $a$ is unit length of the primitive chain which is set equal to the tube diameter. Such displacement along the tube corresponds to that in real space $\left\langle\Delta x^{2}\right\rangle$ through

$$
\left\langle\Delta x^{2}\right\rangle \cong\left\langle\Delta s^{2}\right\rangle^{\nu}
$$

with $\nu=1 / 2$ since the tube is Gaussian. Thus we obtain $\nu_{m}=1 / 4,1 / 2$ for regimes II and III, respectively. In regime IV, the whole chain creeps off the tube over the length scale of $R_{I}$, and motion of the segment coincides with that of the center of mass which follows the linear relation $\phi_{n}(t) \sim R_{I}^{2} t / \tau_{d}$. Here $\tau_{d} \sim M^{3} / a^{2}$ is the reptation time.

In regime IV of a model simulating a Gaussian chain in the regularly distributed obstacles [3], viscosity $\eta$, which is compared to $\tau_{d}$ 11, was measured up to $L=50$ with $L$ being the number of beads and was shown to be proportional to $L^{3.41 \pm 0.14}$ using $L \geq 20$ [16]. Recent study on this model of the most concentrated case clearly showed $\nu_{m}=1 / 4$ in the shorter time region, i.e. regime II (regime I was not seen) [17].

Now let us turn to dynamics of a real chain (with the EVE) in space with fixed obstacles. In regime I, as for a Gaussian chain, inner monomers fluctuate without the tube constraint but with the EVE. In this case, or without obstacles $(a=\infty)$, it is known that $\phi_{M / 2}(t) \propto t^{z}$ at $t \ll \tau_{r}$, where $z=1 /\left(1+\left(1 / 2 \nu_{F}\right)\right)$ with $\nu_{F}=3 /(d+2)$ and $d$ being dimension of the space, and $\tau_{r}$ is the rotational relaxation time for a self-avoiding chain 18].

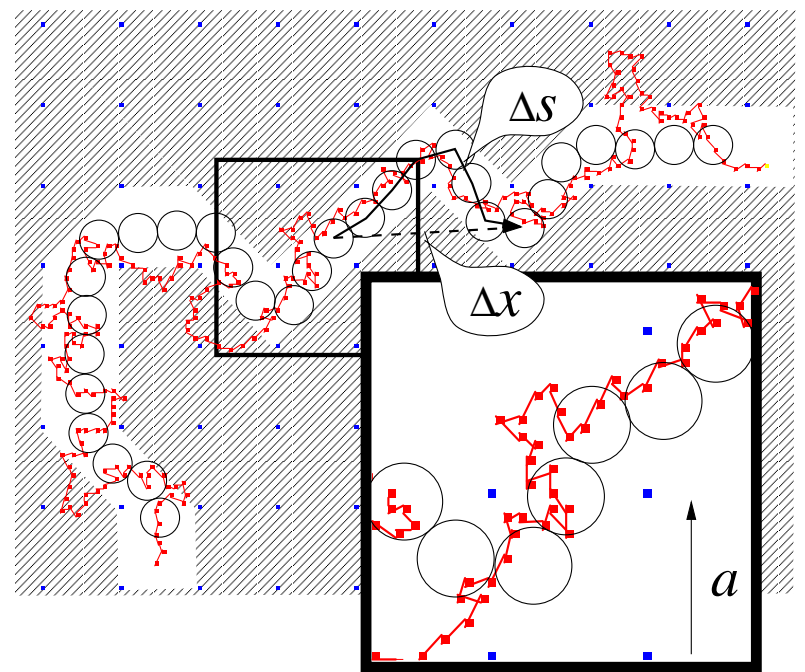

FIG. 2. The schematic representation of a chain of blobs. The polymer configuration drawn is one of instantaneous ones observed in our simulation with $M=400$. The blobs are drawn intuitively from the polymer configurations averaged over time interval of about $\tau_{e}$. In an instantaneous configuration there some local configuration of hernia type are seen.

A polymer configuration in regimes II and III is considered as a chain of blobs along the tube coordinate as shown schematically in Fig. 2. Each blob of an average size $a_{\mathrm{b}}$ consists of $g\left(=a_{\mathrm{b}}^{1 / \nu_{F}}\right)$ monomers on average. In terms of the tube coordinate, the end-to-end distance $R$ is written $R / a_{\mathrm{b}} \sim(M / g)^{\nu_{F}}$, which implies $R_{I} \propto M^{\nu_{F}}$ for the original chain. In fact the latter has been confirmed numerically at least for low concentration of obstacles in two dimensions as shown in Fig. 3. Thus the EVE plays an essential role in determining a "self-avoiding tube" in these regimes.

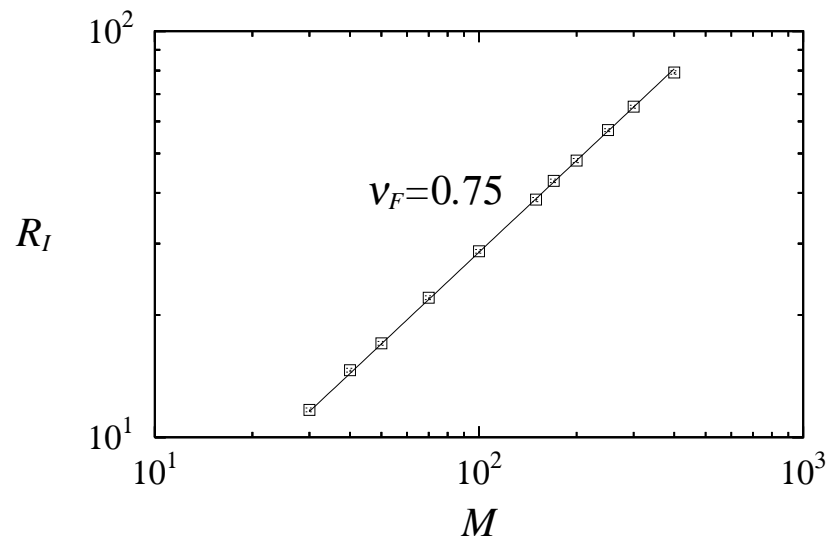

FIG. 3. The relation between radius of gyration $R_{I}$ and length $M$ at $a=20$.

For dynamical aspects under a given polymer configuration (and so a self-avoiding tube), however, the EVE between the blobs of length scale $a_{\mathrm{b}}$ is considered to be screened out 19 21. Accordingly, the diffusion process of blobs along the tube is the same as that of the Gaussian chain [1]. Thus, the exponents of the mean square displacement of the center monomer along the tube $\phi_{M / 2 \|}(t)$ are given by $1 / 2$ and 1 for $t\left\langle\tau_{\theta}\right.$ and $t>\tau_{\theta}$, respectively, where $\tau_{\theta}=\tau_{e}(M / g)^{2}$ is the Rouse relaxation time of the chain of blobs. Now taking into account eq. (11) with $\nu=\nu_{F}$, we obtain the exponents $\nu_{m}=\nu_{F} / 2$ and $\nu_{F}$ in regimes II and III, respectively. In regime IV $\nu_{m}=1$ as in the Gaussian chain.

Now we derive $\phi_{n}(t)$ with $n \sim M / 2$ in each regime more quantitatively. As the Rouse and the reptation theory claim relations $\tau_{\theta} / \tau_{e} \sim[M / g]^{2}$ and $\tau_{d} / \tau_{e} \sim[M / g]^{3}$, the pre-factors of $\phi_{n}(t)$ in the all regimes can be determined by matching its scaling forms in the neighboring regimes of $\tau_{e}, \tau_{\theta}$ and $\tau_{d}$. Setting $b, \zeta / k_{B} T=1$ as before and noting that $\phi_{n} \sim R^{2}\left[t / \tau_{d}\right]$ in regime IV, we obtain $\phi_{n}(t)$ and the cross-over times as listed on Table I.

\begin{tabular}{|c|c|c|}
\hline \hline & real chain & Gaussian chain \\
\hline $\mathrm{I}$ & $\phi_{n} \sim t^{z}$ & $\phi_{n} \sim t^{1 / 2}$ \\
\hline$\tau_{e}$ & $\sim a_{\mathrm{b}}^{2 / z}$ & $\sim a^{4}$ \\
\hline $\mathrm{II}$ & $\phi_{n} \sim a_{\mathrm{b}}^{2}\left[t / \tau_{e}\right]^{\nu / 2}$ & $\phi_{n} \sim a^{2}\left[t / \tau_{e}\right]^{1 / 4}$ \\
\hline$\tau_{\theta}$ & $\sim \tau_{e}[M / g]^{2}$ & $\sim M^{2}$ \\
\hline $\mathrm{III}$ & $\phi_{n} \sim a_{\mathrm{b}} R\left[t / \tau_{\theta}\right]^{\nu}$ & $\phi_{n} \sim a R\left[t / \tau_{\theta}\right]^{1 / 2}$ \\
\hline$\tau_{d}$ & $\sim \tau_{e}[M / g]^{3}$ & $\sim M^{3} / a^{2}$ \\
\hline $\mathrm{IV}$ & $\phi_{n} \sim R^{2}\left[t / \tau_{d}\right]$ & $\phi_{n} \sim R^{2}\left[t / \tau_{d}\right]$ \\
\hline \hline
\end{tabular}

TABLE I. Expressions of $\phi_{n}(t)$ in the four regimes 
Lastly we consider the relaxation time $\tau_{D}$ of the autocorrelation function of the end-to-end vector defined by $P(t)=\langle\mathbf{R}(t) \mathbf{R}(0)\rangle \propto \exp \left[-t / \tau_{D}\right]$. For regime IV the reptation theory 11] predicts that $\tau_{D}$, called the reptation or disengagement time, is obtained from onedimensional diffusion equation of the primitive chain. It is given by $\tau_{D}=L^{2} / \pi^{2} D_{\mathrm{c}}$, where $D_{\mathrm{c}}=1 / M$ is the diffusion constant along the tube, and $L$ is the length of the primitive chain and is given by $a L=M$ for a Gaussian chain ( note we put $k_{B} T / \zeta=1$ and $b=1$ ). We can rewrite $\tau_{D}$ for a self avoiding chain by replacing $L$ by $a_{\mathrm{b}} M / g$. This is because the motion of the chain along the tube is described by dynamics of a sequence of blobs which behave as a Rouse chain. Therefore $\tau_{D}$ and $\tau_{d}$ in Table If are related as

$$
\tau_{D}=\frac{1}{\pi^{2}}\left[\frac{R}{R_{I}}\right]^{3 / \nu_{F}} \tau_{d} \cong 3.65 \tau_{d}
$$

for a real chain.

\section{RESULTS}

In Fig. 4 we show the result of the mean square displacement of the center monomer $\phi_{M / 2}(t)$ obtained from our longest chain $M=400$ in two dimensions. As listed on Table 1 in the previous section, $\phi_{M / 2}(t)$ is expected to exhibit different power-law dependences on $t$ in the four regimes: with $d=2$ and so $\nu_{F}=3 / 4$ and $z=3 / 5$, $\nu_{m}=3 / 5,3 / 8,3 / 4$ and 1 in from regime I to IV, respectively. These theoretical results are well reproduced in our simulated results in the figure. The auxiliary line in the shortest time interval (regime I) stands for the least square fit of our separately simulated data of the $M=400$ chain with $a=\infty$ (no obstacles) to the power-law function of $t$. The exponent is estimated as $\nu_{m}=0.60 \pm 0.01$. The data of $a=20$ lie upon this line at $10 \lesssim t \lesssim 2 \times 10^{3}$. as well.

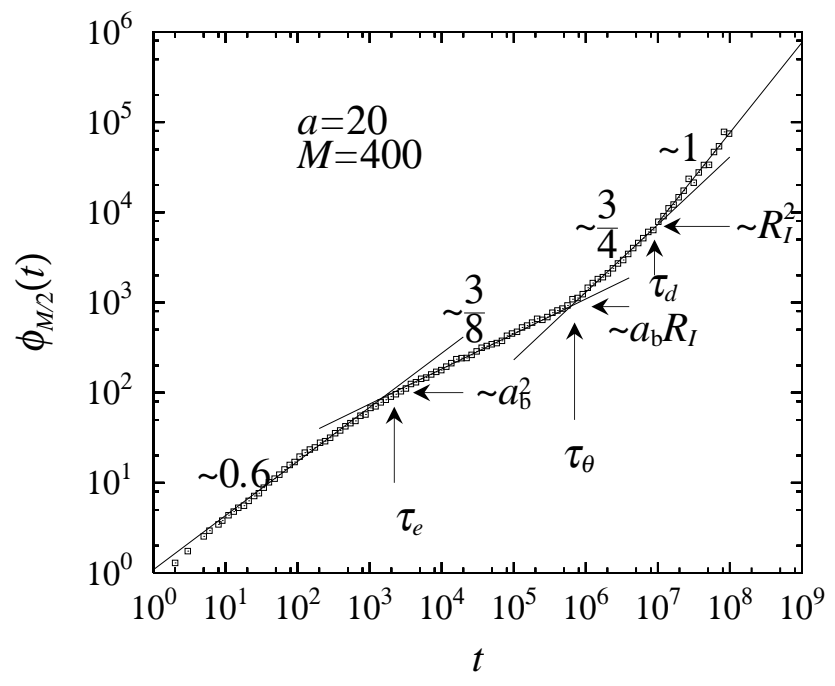

FIG. 4. The mean square displacement of the center monomer of the $M=400$ chain in regular distributed (spacing $a=20$ ) obstacles.

The tube model predicts the crossover time between regimes I and II, where fluctuating monomers start to feel the wall of tube and to form blobs, as $\left(t, \phi_{M / 2}(t)\right)=$ $\left(\tau_{e}, a_{\mathrm{b}}^{2}\right)$. From inspection of the figure, its ordinate seems significantly smaller as compared to $a^{2}$. It is estimated as $a_{\mathrm{b}} \sim a / 2$.

We may think of another diameter $d_{T}$, which corresponds to diameter of a straight tube with hard wall. The mean square displacement of a monomer perpendicular to tube $\phi_{M / 2 \perp}(t)$ for $t \gg \tau_{e}$ is estimated as

$$
\begin{aligned}
\phi_{M / 2 \perp}\left(t \gg \tau_{e}\right) \cong \frac{\sum_{i=1}^{d_{T}-2} \sum_{j=1}^{d_{T}-2}[i-j]^{2}}{\sum_{i=1}^{d_{T}-2} \sum_{j=1}^{d_{T}-2}} \\
=\frac{1}{6}\left[d_{T}-1\right]\left[d_{T}-3\right] .
\end{aligned}
$$

Replacing $\phi_{M / 2 \perp}(t)$ by $\phi_{M / 2}\left(\tau_{e}\right)=a_{\mathrm{b}}^{2}=114 \pm 5$ and substituting it to eq. (3), we get $d_{T} \simeq 28.2 \pm 0.57$. This indicates that the tube diameter $d_{T}$ is bigger than $a$ since the "tube" is not a tube with hard wall but is formed in a space of the point obstacles.

The data in Fig. 4 also demonstrate nicely the chain dynamics in the "self-avoiding tube" described in the previous section. In fact the predicted exponents $3 / 8$ and $3 / 4$ in regimes II and III respectively are ascertained with the appropriate crossover point $\left(t, \phi_{M / 2}(t)\right)=$ $\left(\tau_{\theta}, a_{\mathrm{b}} R_{I}\right)$. Finally at $t \gtrsim \tau_{d}$ crossover from region III to IV occurs, and the linear relation $\phi_{M / 2}(t) \sim t$ is reproduced in regime IV.

In order to confirm further the compatibility of theoretical arguments on the polymer dynamics in the selfavoiding tube, we have carried out scaling analyses on $\phi_{M / 2}(t)$ with different $M(=100 \sim 400)$. Some raw data are shown in Fig. 5 . 


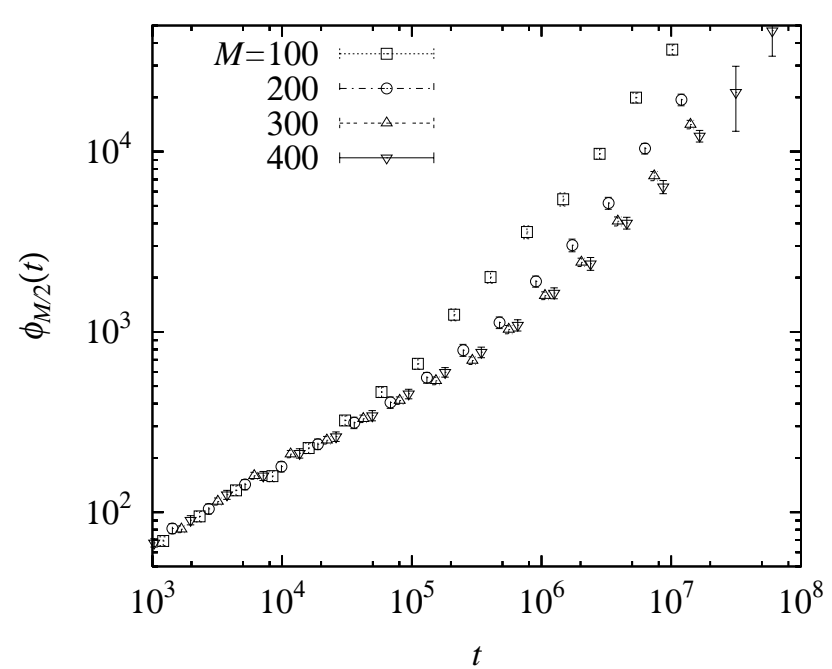

FIG. 5. The simulated data of $\phi_{M / 2}(t)$ with $M=100$, 200, 300 and 400 at $a=20$. As expected from the fact $R_{I}>a_{\mathrm{b}}$ for these $M$ they coincide with each others in regime I and a shorter time range of regime II.

In Fig. 6, double logarithmic plots of $\phi_{M / 2}\left(t / \tau_{\theta}\right) / a_{\mathrm{b}} R_{I}$ in terms of $t / \tau_{\theta}$ around crossover between regimes II and III are shown for $M=100,200,300$ and 400. Here the value $\tau_{\theta}$ is determined as $\tau_{\theta}=\tau_{e}\left[R_{I} / a_{\mathrm{b}}\right]^{2 / \nu_{F}}$, using $R_{I}$ and $\nu_{F}$ in Fig. 3 and $\tau_{e}$ and $a_{\mathrm{b}}$ from Fig. 5 . It can been seen that the scaling fit is satisfactory for $M \geq$ 200. The auxiliary lines fitted to the data yield exponents $0.38 \pm 0.01$ and $0.73 \pm 0.02$ in the regimes II and III, respectively, and they cross at the point $(1,1)$ within our numerical accuracy. The data of the shortest $M=100$ chain, on the other hand, deviates from the others in early time region $t / \tau_{\theta} \lesssim 2 \times 10^{-1}$, where it is in the regime I. So it can be said that dynamics in regimes II and III for $M \geq 200$ is interpreted as monomer motion through blobs along the self-avoiding tube.

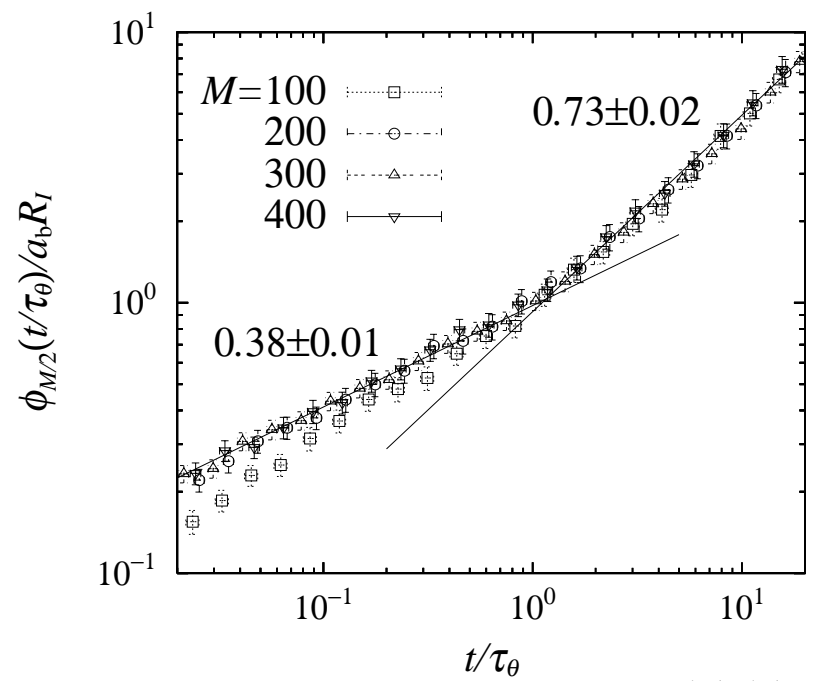

FIG. 6. The double logarithmic plot of $\phi_{M / 2}\left(t / \tau_{\theta}\right) / a_{\mathrm{b}} R_{I}$ with $t / \tau_{\theta}$.
We show double logarithmic plots of $\phi_{M / 2}\left(t / \tau_{d}\right) / R_{I}^{2}$ against $t / \tau_{d}$ in regimes III and IV in Fig. 7. Here $\tau_{d}=$ $\tau_{e}\left[R_{I} / a_{\mathrm{b}}\right]^{3 / \nu_{F}}\left(\propto M^{3}\right)$. The data in each regime are scaled to a single curve for $M \geq 200$, and they cross near $(1,1)$ as well except for the shortest $(M=100)$ chain. This result supports the crossover scenario from the reptation regime of III to the over-all diffusion regime of IV.

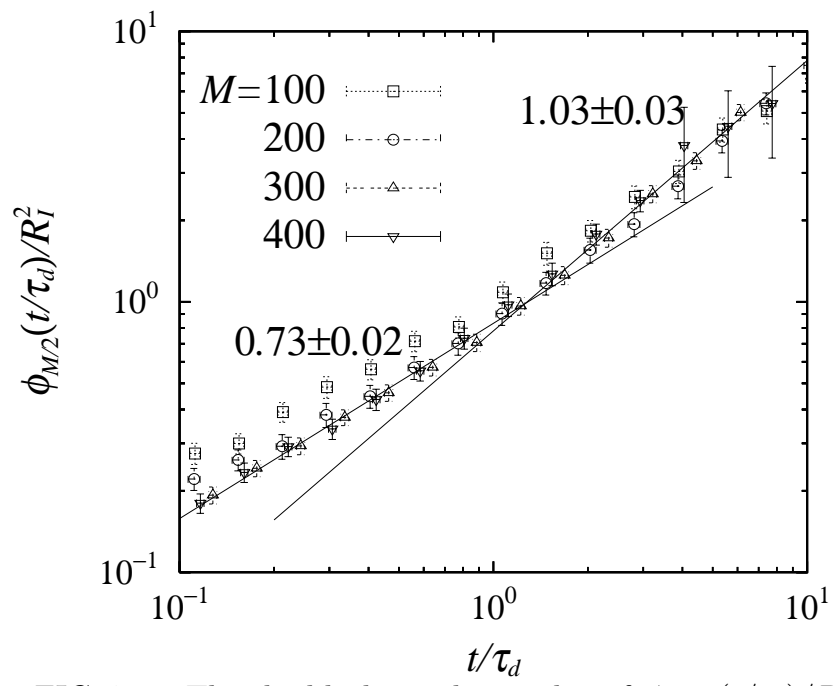

FIG. 7. The double logarithmic plot of $\phi_{M / 2}\left(t / \tau_{d}\right) / R_{I}^{2}$ with $t / \tau_{d}$.

Next we discuss behavior of some other quantities than $\phi_{M / 2}(t)$ mainly in regime IV. Figure 8 shows the semi$\log$ plot of $P(t)$ for $M=50 \sim 400$. At long time regions, which corresponds to regime IV with $\phi_{M / 2}(t) \propto t, P(t)$ of each $M$ is well fitted to an exponential function. The plots of the relaxation time extracted from $P(t)$ and the one evaluated by means of the r.h.s. of eq. (2) against $M$ are shown in Fig. 9. The two estimates of $\tau_{D}$ coincide with each other even up to numerical coefficient and clearly display the slope 3 within error of $3 \%$, i.e. $3.0 \pm 0.1$, for $M \geq 70$. The result strongly supports the argument that the primitive chain, whose unit length is identical to blob size $a_{\mathrm{b}}$, diffuse along itself.

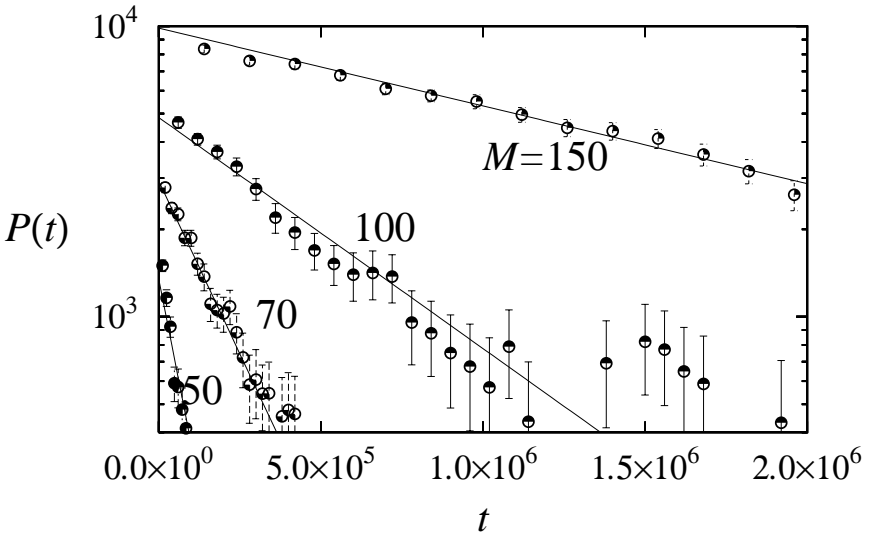




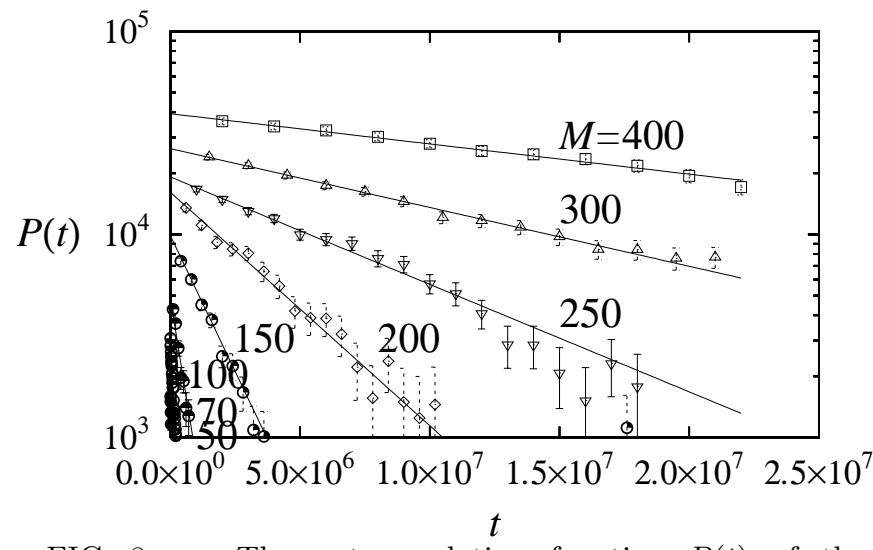

FIG. 8. The autocorrelation function $P(t)$ of the end-to-end vector observed for each lengths.

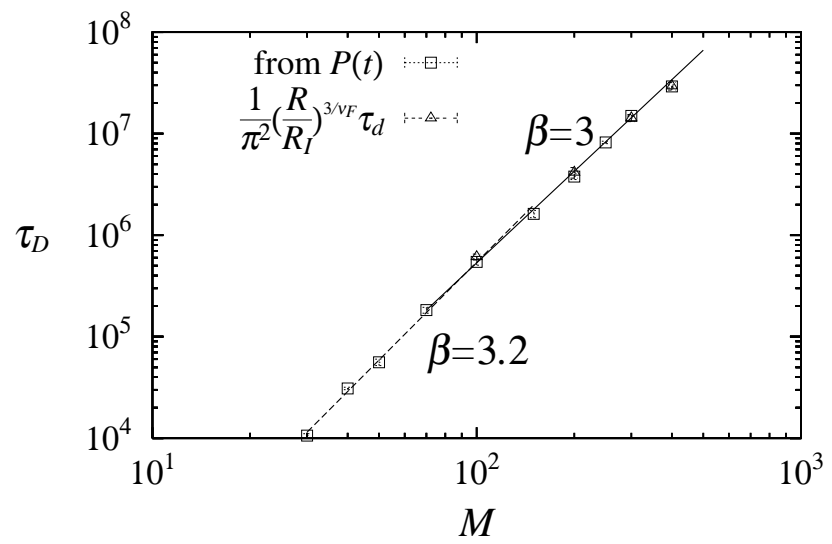

FIG. 9. The rotational relaxation times $\tau_{D}$ obtained from Fig. 8 and $\frac{1}{\pi^{2}}\left[\frac{R}{R_{I}}\right]^{\nu_{F} / 3} \tau_{d}$ of eq. (2).

In such a free diffusion regime monomer motion is identical to that of the center of mass. The pre-factor of linear fit of the mean square displacement of the center of mass $\phi_{\text {c.m. }}(t)$ in the longest time range (which includes regime IV) gives us diffusion constant $D_{G}$ [11. Typical data of $\phi_{\text {c.m. }}(t)$ are shown in Fig. 10 . We have determined $D_{G}$ by the linear fit of the data in $t \gtrsim \tau_{d}$, and plotted them against $M$ in the inset of Fig. 10 .

The exponent $\alpha$ for $D_{G} \sim M^{\alpha}$ is estimated as $\alpha \sim$ -1.5 for large $M$. It is different from what Gaussian tube theory predicts $(\alpha \sim-2)$. It is, instead, readily understood if we plot $\tau_{d}\left(\propto M^{\beta}\right)$ and $R_{I}^{2} / D_{G}\left(\propto M^{2 \nu-\alpha}\right.$ ) against $M$ simultaneously. As seen in Fig. 11 the scaling relation $\beta=2 \nu-\alpha$ holds as is derived from scaling argument of the reptation theory 21 .

We have obtained $\alpha \sim-1.5$ using the data with $M=70 \sim 400$. When the data with $M=30 \sim 150$ are used, on the other hand, it becomes $\alpha \sim-1.7$. This is consistent with the results obtained in our previous work [10] and is quantitatively in agreement with other results such as reported in [16]. Such a large magnitude of $\alpha$ obtained from smaller $M$ may be attributed to tubelength fluctuation which gives rise to a leading correction to the scaling described so far. Actually we have confirmed $\beta=3.2 \pm 0.1$ from the data with $M=30 \sim 150$ as seen in Fig. 9. Then the scaling relation $\beta=2 \nu-\alpha$ still holds for these exponents as shown in Fig. 11.

Now we taking into account such correction, the deviation of the $M=100$ data from the scaling curve in Fig. 7 is naturally interpreted that $M=100$ is in the crossover range of the parameter space of $M$.

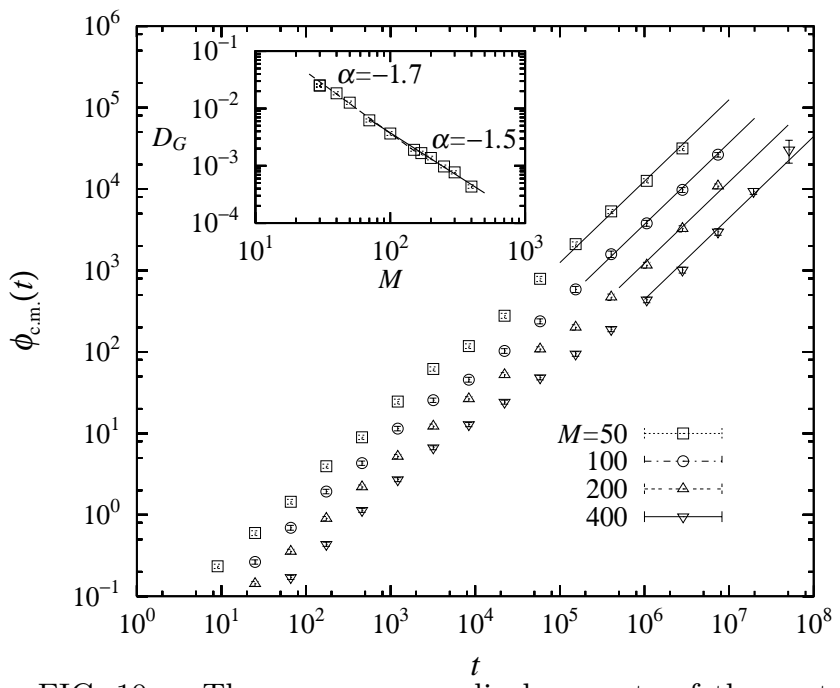

FIG. 10. The mean square displacements of the center of mass $\phi_{\text {c.m. }}(t)$ and their diffusion constants $D_{G}$, calculated from tangent of final linear regime, are plotted against $M$.

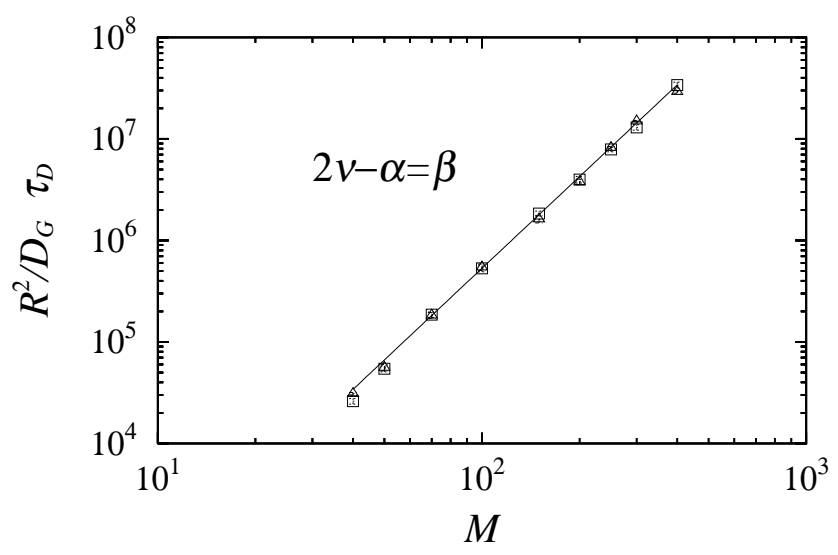

FIG. 11. The scaling relation $\beta=2 \nu-\alpha$.

\section{CONCLUSIONS}

We investigated on the dynamics of single long selfavoiding polymer in the regularly distributed [spacing $a=20$, i.e. concentration $c=1 \%$ ] obstacles in $2 \mathrm{D}$ by the e-BFM and analyzed the results especially in connection with the tube model. The following four regimes are observed in the mean square displacement of center monomer $\phi_{M / 2}(t) \propto t^{\nu_{m}}$ as $\nu_{m} \sim 0.6,3 / 8,3 / 4$ and 
1. These exponents are explained by the "self-avoiding tube" model. That is, the defect dynamics through blobs along such a swelled tube give the exponent of $\nu_{m}$ as $3 / 8$ and $3 / 4$ at the second and the third regimes respectively. We have shown the validity of such argument through the scaling analyses on the crossover regions and the free diffusion regime.

\section{ACKNOWLEDGMENTS}

The computation in this work has been done using the facilities of the Supercomputer Center, Institute for Solid State Physics, University of Tokyo, and those of the Computer Center of University of Tokyo.

[1] P. G. de Gennes, J. Chem Phys. 55, 572 (1971).

[2] A. Baumgärtner and K. Binder, J. Chem. Phys. 75, 2994 (1981).

[3] K. E. Evans and S. F. Edwards, J. Chem. Soc. Faraday Trans. 2 77, 1891 (1981).

[4] B. Norden, C. Elvingston, M. Jonsson, and B. Åkerman, Quart. Rev. Biophys. 24, 103 (1991).

[5] B. H. Zimm and S. D. Levene, Quart. Rev. Biophys. 25, 171 (1992).

[6] R. H. Austin et al., Physics Today 50, 32 (1997).

[7] M. Yanagida, Y. Hiraoka, and I. Katsura, Cold Spring Harbor Symp. quant. Biol. 47, 1982 (1982).

[8] C. Bustamante, Annu. Rev. Biophys. Biophys. Chem. 20, 415 (1991).

[9] B. Maier and J. O. Rädler, Phys. Rev. Lett. 82, 1911 (1999).

[10] R. Azuma and H. Takayama, Phys. Rev. E 59, 650 (1999).

[11] M. Doi and S. F. Edwards, The theory of polymer dynamics (Clarendon Press, Oxford, 1986).

[12] I. Carmesin and K. Kremer, Macromolecules 21, 2819 (1988).

[13] I. Carmesin and K. Kremer, in Polymer Motion in Dense Systems, edited by D. Richter and T. Springer (SpringerVerlag, Berlin, Germany, 1988), pp. 214-219.

[14] J. Batoulis, N. Pistoor, K. Kremer, and H. L. Frisch, Electrophoresis 442 (1989).

[15] P. G. de Gennes, Physics 3, 37 (1967).

[16] J. M. Deutsch and T. L. Madden, J. Chem. Phys. 91, 3252 (1989).

[17] U. Ebert, A. Baumgärtner, and L. Schäfer, Phys. Rev. Lett. 78, 1592 (1997).

[18] K. Kremer and K. Binder, J. Chem. Phys. 81, 6381 (1984).

[19] P. Pincus, Macromolecules 9, 386 (1976).

[20] F. Brochard and P. G. de Gennes, J. Chem Phys. 67, 52 (1977).
[21] P.-G. de Gennes, Scaling Concepts in polymer physics (Cornell University Press, Ithaca, New York, 1979). 